\title{
A Review on Reactive and Proactive Wireless Sensor Networks Protocols
}

\author{
Navneet Kaur \\ Research Scholar \\ Deptt of Computer Science and Engineering \\ D.A.V.I.E.T Jalandhar, \\ Punjab, India.
}

\author{
Manjinder Singh Kahlon \\ Asstt. Professor \\ Deptt of Computer Science and Engineering \\ D.A.V.I.E.T Jalandhar, \\ Punjab, India.
}

\begin{abstract}
WSNs are becoming popular in real world applications. Due to the features of the resource-constrained and battery aware sensors; in WSNs energy utilization has found to be a major interesting subject of research. WSNs compose batterypowered nodes which are connected with the base station to for certain action or task. As sensor nodes are battery-powered i.e. will become dead after the consumption of the battery which is also called lifetime of WSNs. So using the energy in well-organized way may result in prolonging the lifetime of the WSNs. This paper has evaluated and explores the various stable election based protocols to find the short coming of the earlier work in heterogeneous WSNs.
\end{abstract}

\section{Keywords:}

Wireless Sensor Networks, Stable Election Protocol, Lifetime, Hybrid Energy Efficient Reactive Protocol, Cluster Head, Low Energy Adaptive Clustering Hierarchy, Stable Cluster Head Election Protocol.

\section{INTRODUCTION}

Technology has advanced to an extent such that it has moved from wired to wireless domain. The wireless devices are functionally depending upon their battery life. Wireless communication technologies are continuously growing in diverse areas and provide new and better opportunities for different business environments. Recent development in wireless communication and electronics have empowered the deployment of small, relatively in-expensive and lowpower devices called micro sensors that may be connected by a wireless network. These wireless micro sensor networks present a new standard for obtaining data from the surrounding environment and also allow the reliable examining of a number of environments for various applications.

\section{ORIGIN OF WIRELESS SENSOR NETWORKS $\left(\mathbf{W S N}_{\mathrm{s}}\right)$}

The origin of wireless sensor networks traces back to the cold war era where a system of acoustic sensors on the ocean bottom was placed by USA military in 1950 for the sound surveillance to detect and track Soviet submarines. At the same time, US developed the method of air defence radars to defend its territory.

Echoing the investments created in Nineteen Sixties and Seventies to develop the hardware for today's net , the U.S Defence advanced analysis comes agency (DARPA) started Distributed sensing element Network (DSN) program in 1978 to formally explore the challenges in implementing distributed / wireless sensing element networks. With the emergence of DSN and its progress into academia, WSN technology soon found its way into academia and scientific research. Governments and universities began using WSNs in many applications such as forest fire detection, air quality monitoring, and natural disaster prevention.

While the commercial demand for WSNs was strong, moving beyond these applications was a challenge. The early military, science/technology were all based on heavy, expensive sensors and possessory networking protocols. These WSNs increased the functionality but other factors such as hardware cost, deployment cost, power consumption, scalability and networking standards were not considered. Reducing WSN deployment costs while increasing functionality involves major advances in sensors, CMOS based semi-conductor devices, networking protocols and energy storage or generation technology.

The advancement in semi-conductor, networking and material science technologies are driving the deployment of large-scale WSNs. Together, these technologies have made up to create a new generation of WSNs that are different from the WSNs developed and deployed 5 to 10 years ago. Today's WSNs have low deployment and maintenance costs, last longer and are more rigorous. Recent advances in sensing, computing and communication technologies coupled with the need to continuously monitor physical phenomenon have led to the development of WSNs.

\section{SIGNIFICANCE OF WSNs}

Wireless sensor networks have gained so much importance over the past few years because of the many applications in which the sensors can be used to monitor and control different physical phenomenon. WSNs are among the most widely used ad-hoc wireless networks. WSNs are referred as infrastructure less networks that are capable of wireless communication. Recent technological advancements have enabled the development of relatively small, inexpensive wireless sensors that consume low power. These sensors can be deployed at a cost much less than those traditional wired systems.

Wireless sensor network is a small system that is able to communicate over short distances. This small system is made up of sensor nodes. Sensor nodes can be imagined as small computers, extremely basic in terms of their interfaces and their components. They usually consist of a processing unit with limited computational power and limited memory, sensors or MEMS (including specific conditioning circuitry), a communication device (usually radio transceivers or 
alternatively optical), and a power source usually in the form of a battery. It is a collection of hundreds and thousands of sensor nodes that are organized into a co-operative network.

These nodes are adaptive and self-organized and integrate the functions of collecting data, processing it and then communicating it to the other nodes in the network. These nodes work together to form a powerful sensor network. The sensing elements sense data from the environment, the processing system performs computation on the sensed data and the communication or radio system exchange the information with other nodes in the system. These sensors can detect several environmental events such forest fire, wind velocity, humidity, and soil temperature. This information can then be transmitted to a base station (BS) or sink.

\section{A. Characteristics of WSNs:}

- Power consumption constraints for nodes using batteries or energy harvesting

- Ability to cope with node failures

- Mobility of nodes

- Communication failures

- Heterogeneity of nodes

- Scalability to large scale of deployment

- Ability to withstand harsh environmental conditions

- Ease of use

Nodes can be stationary or mobile. If mobile, then they are evenly distributed over the sensing field. And if stationary, then they use a deployment mechanism. In most of the cases, sensor nodes have non-rechargeable battery that cannot be replaced. The energy of the nodes is consumed during sensing phase first and later during the transmission and reception of data. So it is practically impossible to recharge or replace the battery of node once it has been deployed. WSNs are based on energy-constrained, battery-powered devices and in case large networks are needed to function for a longer period of time, balanced energy consumption is a critical issue.

To extend the lifetime of the network, efficient use of available energy is the subject to be considered. WSN lifetime is the time interval between the start of the first transmission until the expiry of the last alive node i.e. the time when the operation starts until the percentage of the sensor nodes fall below a specific threshold. Since, most of the energy is consumed due to communication, so an efficient energy saving routing protocol is required to extend the survival rate or the network lifetime. Routing protocols have the capability to reduce the energy consumption of WSNs by choosing minimal routes. Among many routing protocols, the most effective is the cluster-based technique.

In cluster-based routing, the sensor nodes are grouped together into a number of clusters and the nodes of the cluster send their data to a specific node called cluster head $(\mathrm{CH})$. The cluster head $(\mathrm{CH})$ is present in each cluster and is responsible for collecting the data from the other nodes. After gathering all the data from sensor nodes, the $\mathrm{CH}$ then transmits it to the sink or Base station (BS) and these local Base Stations transmit the data to the global BS, where it is accessed by the end user.

The base stations are one or more components of the WSN with much more computational, energy and communication resources. They act as a gateway between sensor nodes and the end user as they typically forward data from the WSN on to a server. The cluster heads are selected based upon a specific criterion. Since $\mathrm{CHs}$ are an important part of clusterbased routing protocols, the $\mathrm{CH}$ selection affects the network lifetime, energy consumption rate and the delay occurred in the delivery of packets.

\section{ROUTING PROTOCOLS}

We can categorize routing protocols based on the areas in which they are used and that can be:

(a) Proactive routing protocols (table-driven): These are used where information about the nodes is continuously required. The nodes sense the network on a regular basis and communicate the data sensed to the other nodes. Every sensor node in the network has a routing table which keeps record of all the destinations and the number of hops that are need to reach the destination. A sequence number is generated by the destination each time the data is broadcast. Each time the cluster changes, and once the cluster-heads are selected, the $\mathrm{CH}$ broadcasts the report time and attributes to the network.

Report time is the time interval between the successive reports sent by a node. At each report time, the cluster nodes sense the parameters defined in the attributes and send the data to the $\mathrm{CH}$. The $\mathrm{CH}$ then aggregates the data

and communicates it to the BS. In proactive networks, the nodes periodically switch on their sensors, sense the environment and communicate the data to the other nodes. Thus they provide a continuous report of the parameters. These protocols are suitable for the networks which have fewer number of nodes a they have to keep updating the routing table entries for each node.

E.g. Destination Sequence Distance Vector (DSDV)

(b) Reactive routing protocols (on-demand): They are quite similar to the former protocols but differ in the transmission time of the data to other nodes. Here, the constant update of routing table is not needed. They search for the route when needed i.e. only when they find a drastic change in the value of the sensed attributes. And when found a route, they establish a connection to transmit data from source to destination.

Each time the cluster changes, the $\mathrm{CH}$ broadcasts the hard threshold $\left(H_{T}\right)$ and soft threshold $\left(S_{T}\right)$ to the other members of the cluster. $\left(H_{T}\right)$ is then absolute value of the attribute exceeding which, the node must switch on its transmitter and report it to the $\mathrm{CH} .\left(S_{T}\right)$ is the small change in the value of the sensed attribute that initiates the node to switch on its transmitter and transmit.

The first time when a parameter reaches its $\left(H_{T}\right)$, the node senses it and transmits it. The sensed value is stored in a node called sensed value (SV). The nodes will next send the data in the current period only

When the current value of sensed attribute is greater than $\left(H_{T}\right)$ and differs from SV by an amount equal or greater than $\left(S_{T}\right)$.

E.g. AODV (Ad-hoc On-demand Distance Routing)

As WSNs have resource constraints like limited energy, so an effective energy consumption protocols is desired to fulfil the energy needs of the network. Following are some recent cluster-based energy saving protocols: 


\section{Performance Measures:}

Following are the measures that are used to evaluate the performance of clustering protocols:

- Stability period: time interval from start of operation until death of first node

- Instability period: time interval from death of first node until death of last node

- Network lifetime: time interval from start of operation to death of last alive node

- Number of CHs per round

- Number of alive nodes per round

(a) Low Energy Adaptive Clustering Hierarchy (LEACH)

WSNs are micro sensor systems that are spatially distributed. WSN is a power constrained system as the sensor nodes have limited battery life that shortens the network lifetime. Maximizing the network lifetime depends upon an efficient communication protocol. Energy consumption is, therefore, a critical design issue in WSN. A cluster-based technique is the basic method to increase the scalability, performance, efficiency and lifetime of the network.

LEACH[11], a hierarchical clustering routing protocol, was proposed by Chandrakasan, Heinzelman and Balakrishnan, in MIT. Leach is a protocol that works well in homogenous networks. In a homogenous network, all nodes have equal amount of initial energy. Basically there are two types of routing protocols in WSNs: Flat routing protocols are those in which the routing condition of each node in the network is the same. There are no special nodes in network and each node has equal status. So, the network traffic is distributed equally among all nodes. Comparatively, hierarchical routing protocols make use of the concept of clusters that divides all nodes into groups or clusters. Nodes in this type of network have different levels. A CH is selected among all the nodes and different hierarchical routing protocols may use different methods of selecting CHs.

LEACH is a low energy protocol that may adapt clustering. It is a cluster-based protocol that utilizes the concept of randomized rotation of local cluster-heads and distributes the energy load evenly among all the sensor nodes in the sensing field of the network.

\section{Characteristics of LEACH:}

- Set up of clusters through local collaboration and control

- $\quad$ To reduce the Data aggregation in network traffic

- Local compression to scale back world communication.

- Randomized rotation of the cluster heads and also the corresponding clusters.

- $\quad$ Random Death of nodes

Assumptions of LEACH:

- All nodes are similar in context of initial energy.

- $\quad$ All nodes make use of Omni-directional antenna

- $\quad$ BS is fixed and is distant from WSN
- $\quad$ Energy consumption of each node to send data to other is equal.

LEACH uses the concept of rounds. The work period id referred as a round. Each round constitutes 2 phases: (a) Setup phase, (b) Steady phase

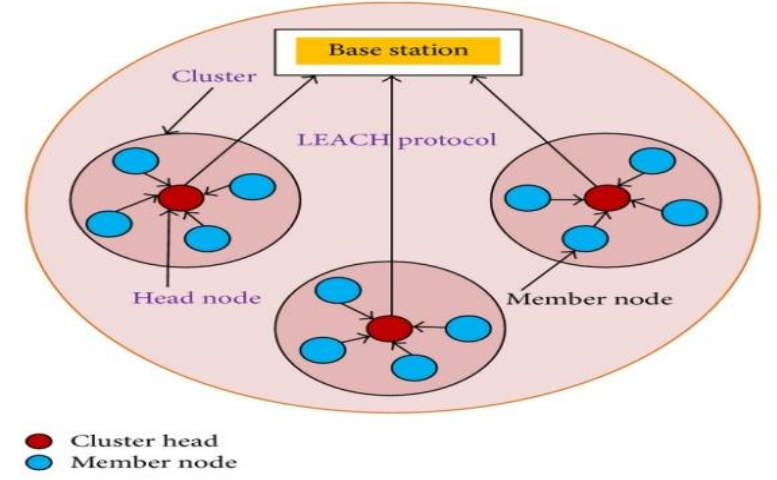

Figure 2. LEACH protocol (www.hindawi.com/journals)

Setup Phase: In the foundation or setup phase, each node decides whether to become a cluster-head $(\mathrm{CH})$ in the present round. To be selected a $\mathrm{CH}$, each node generates a random number between 0 and 1 . The threshold is set up for the current round and the generated random number is compared to the threshold of the given round. If the number is less than threshold $\mathrm{T}(\mathrm{n})$, that particular node will be selected the $\mathrm{CH}$ for that round.

The threshold is set up as:

$\mathrm{T}(\mathrm{n})=\left\{\begin{array}{l}\frac{\mathrm{p}}{1-\mathrm{p} *\left(\operatorname{rmod} \frac{1}{p}\right)} \\ 0, \quad \text { otherwise }\end{array}, \mathrm{n} \in \mathrm{G}\right.$

Here $\mathrm{p}$ is the ratio of cluster-head nodes in the total number of nodes.

$r$ is the current round number. $G$ is the set of nodes that have not been selected as $\mathrm{CH}$ nodes in the former $\frac{1}{\mathrm{p}}$ rounds.

The high energy cluster head position rotates among the various sensors in order to not to drain the battery of a single sensor. Sensors elect themselves to be the local cluster heads at any given time with a certain probability, and broadcast their status to other sensors each sensor node choosing the cluster-head with strongest signal. Each node takes the decision independent of the other nodes to become cluster head. Then cluster head creates a TDMA schedule for all nodes within its cluster telling each node when it can transmit. It allows radio component of each non cluster head to be turned off at all times except during its transitions time, thus minimizing the energy dissipation in the individual sensors and their receivers on during set up phase to hear the advertisements of all cluster heads.

Steady phase: this operation is divided into frames where the nodes send their data to $\mathrm{CH}$ at most one frame during their transmission time slot. The $\mathrm{CH}$ transmits the aggregated data to the Base station (BS).

This protocol selects the $\mathrm{CH}$ randomly and the total energy load of the network is equally distributed to each sensor node by which it can consume less energy and thereby improving the network lifetime. LEACH outperforms conventional routing protocols like direct transmission, minimum- 
transmission-energy, in the static clustering algorithms. LEACH is distributed and nodes do not require control information from the base station nor knowledge of the global network.

The LEACH protocol carries out energy optimization and also reduces the amount of data transmitted to prolong the network lifetime. However, it has some drawbacks:

- It may happen that the same $\mathrm{CH}$ node is selected $\mathrm{CH}$ again in some other round as it has more energy

- It has a hotspot problem, i.e. the $\mathrm{CH}$ uses more energy than normal sensor nodes

- The normal sensor nodes that overlap their sensing fields generate duplicate data that create

\section{Unnecessary load on other $\mathrm{CHs}$}

- The CHs are selected randomly, so if the node with low energy is selected $\mathrm{CH}$, then that node may be heavily loaded and more energy will be consumed that results in early death of these nodes and ultimately reduce the network lifetime.

\section{(b)Stable Cluster Head Election (SCHE) Protocol}

It is based on LEACH architecture that uses clustering technique. Its goal is to reduce the energy consumption of each sensor node and thus minimizing the overall energy dissipation of the network. SCHE is a source driven protocol based on timely reporting. So the sensor node will always have some data to transmit to the Base station. It also makes use of data aggregation to avoid information overload.

It provides $\mathrm{n}$ analytical framework to attain the stable probability for a node to be a cluster-head to minimize energy consumption. It is necessary to apply suitable $\mathrm{CH}$ election mechanism to minimize energy consumption of each sensor node that ultimately results in reduced energy dissipation. SCHE was proposed where this mechanism was applied by obtaining the optimum value of probability for a node to become a $\mathrm{CH}$ and consumes significantly less energy compared to LEACH. It also reduces consumption by minimizing distance between $\mathrm{CH}$ and $\mathrm{BS}$.

\section{(c) Stable Election Protocol (SEP)[9]}

There are some drawbacks associated with LEACH such as: single hop routing is used where each node can transmit directly to $\mathrm{CH}$ and sink. $\mathrm{CHs}$ are elected randomly. Therefore there is a possibility that all $\mathrm{CHs}$ will be concentrated in the same area. The concept of dynamic clustering is used which leads to unnecessary overhead due to cluster changes. The protocol also assumes that all nodes have amount of energy for each node.

But recent protocols like SEP is opposite to that of LEACH as it considers energy heterogeneity where the factors mentioned are just a possibility. WSNs have assumed homogenous nodes for most of the time. But these nodes also differ in initial amount of energy and also in depletion rate. This leads to the heterogeneous networks where we consider two or more types of nodes. SEP is proposed for two-level heterogeneous networks that has two types of nodes according to their initial energy. The nodes that have higher amount of energy than the other nodes are called advance nodes and the other nodes are the normal nodes.

In SEP the election probabilities of nodes are weighted by the initial energy of each node to become the cluster-head relative to the other nodes in a network. This prolongs the time period before the death of first node in the system. SEP approach makes sure that $\mathrm{CH}$ election is done randomly and is distributed based on the energy of each node assuring the uniform utilization of the nodes energy. SEP consists of advance nodes that carry more energy than the normal nodes at the beginning, so it enhances the stability period of the network.

Normal nodes have initial energy $E_{0}$, and advance nodes have initial energy $(1+a) E_{0}$. Where (a) is the percentage of energy higher than normal nodes. Each node has a probability to become a $\mathrm{CH}$ and each node generates a random number between 0 and 1 just like in LEACH. If the number is less than threshold $\mathrm{T}(\mathrm{s})$, then that node becomes $\mathrm{CH}$ in the current round. With increase in number of rounds, the $\mathrm{T}(\mathrm{s})$ also increases and reaches 1 only in the last round.

Let $p_{\text {nrm }}$ be the weighted election probability of normal nodes and $P_{a d v}$ be the weighted election probability of advance nodes. Optimum probability of each node to become $\mathrm{CH}$ can be calculated by:

$P_{\text {nrm }}=\frac{P_{\text {opt }}}{1+a m}$
$P_{a d v}=\frac{P_{o p t}}{1+a m} *(1+a)$

' $m$ ' denotes the fraction of advance nodes and ' $a$ ' is the additional energy factor between advance and normal nodes.

The threshold is given by the formula:

$T_{\text {nrm }}=\left\{\begin{array}{c}\frac{p_{\text {nnm }}}{1-p_{\text {nnm }}\left[\text { rmod } \frac{1}{\text { Vnrm }}\right]} \\ 0 \text { otherwise }\end{array}\right.$ if $n_{\text {nrm }} \in \mathrm{G}$

$T_{a d v}=\left\{\begin{array}{c}\frac{P_{a d v}}{1-P_{a d v}\left[r_{\text {mod }} \frac{1}{V_{a d v}}\right]} \\ 0 \text { otherwise }\end{array} \quad\right.$ if $n_{a d v} \in \mathrm{G}$

The total energy of new heterogeneous setting will be:

n. $(1-m) \cdot E_{0}+\mathrm{n} \cdot m \cdot E_{0} \cdot(1+a)=\mathrm{n} \cdot E_{0} \cdot(1+a \cdot m)$

so the total energy of the system is increased by $(1+a . m)$ times.

In order to optimize the stable region of the system the new epoch must become $\frac{1}{p_{o p t}} .(1+a . m)$ as the system has a.m times more energy and $a . m$ times more nodes.

\section{(d)Extended Stable Election Protocol (ESEP)[7]}

It is a modified SEP protocol. Instead of two types of nodes, it considers three nodes based on their energy levels. These nodes are: normal, moderate and advance nodes. The goal of ESEP is to achieve a WSN that maximizes the network lifetime and stability period. Also it must reduce the communication cost and deployment cost. The operation to become a $\mathrm{CH}$ is same as in SEP by generating a random number and then comparing it with the threshold. In ESEP the moderate or intermediate nodes are selected in two ways either by the relative distance of advance nodes to normal nodes or by the threshold of energy level between advance nodes and normal nodes.

The weighted election probabilities are given by: 


$$
\begin{aligned}
& P_{\text {nrm }}=\frac{p_{\text {opt }}}{1+p_{1} \cdot a+k \cdot b} \\
& P_{\text {mod }}=\frac{p_{\text {opt }}}{1+p_{1} a+k \cdot b} \cdot(1+a) \\
& P_{a d v}=\frac{p_{o p t}}{1+p_{1} a+k \cdot b} \cdot(1+b)
\end{aligned}
$$

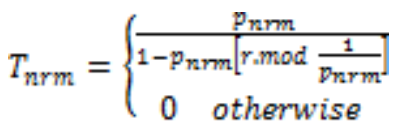

$$
\text { if } n_{n m m} \in G^{\prime}
$$

$$
T_{\text {nrm }}=\left\{\begin{array}{l}
\frac{p_{\text {int }}}{1-p_{\text {int }}\left[\operatorname{rmod} \frac{1}{p_{\text {int }}}\right]} \\
0 \text { otherwise }
\end{array}\right.
$$$$
\text { if } n_{\text {nrm }} \in G^{\prime}
$$

$T_{\text {adv }}=\left\{\begin{array}{c}\frac{P_{a d v}}{1-p_{a d v}\left[P \bmod \frac{1}{V_{a d v}}\right]} \\ 0 \text { otherwise }\end{array}\right.$

$$
\text { if } n_{a d v} \in G^{\prime},
$$

Where G', G' and G'" are the set of normal, intermediate and advance nodes that have not been elected the $\mathrm{CH}$.

Average number of $\mathrm{CHs}$ per round will be:

$n \cdot(1-m-b) \cdot p_{\text {nrm }}+n \cdot b \cdot p_{\text {int }}+n \cdot m \cdot p_{a d v}=n \cdot p_{o p t}$

This shows that due to energy heterogeneity, the energy dissipation is reduced in the network. At the start of each round, the process of cluster change takes place in which the $\mathrm{CH}$ broadcasts some parameters to the other nodes. These parameters are the report time, attributes, soft threshold (ST) and hard threshold (HT). the nodes keep sensing the environment for change in the values of attributes. When the values of attribute set reaches the hard threshold, the radio is turned on and the data is transmitted to the $\mathrm{CH}$. This sensed value is stored in a variable called Sensed Value (SV). The next time the nodes transmit data only when the sensed value is greater than the hard threshold or if the difference between the sensed value and the value in $\mathrm{SV}$ is equal to or greater than the soft threshold. So, these thresholds reduce the number of transmissions as the data will be transmitted only when it reaches hard threshold. Thus, this additional feature of three heterogeneity levels results in increased stability period and network lifetime.

(f)Hierarchical based stable election protocol (HSEP)[3] It is the next level protocol that was proposed after ESEP. The increasing the distance between the $\mathrm{CH}$ and the BS results in increasing the transmission energy because most of the energy is consumed in the transmission process. HSEP is proposed which aims at reducing the transmission energy between the $\mathrm{CH}$ and BS. It brings into consideration the clustering hierarchy which lowers the transmission cost and hence the energy. In this type of clustering we use two types of clusterheads: primary $\mathrm{CHs}$ and secondary $\mathrm{CHs}$.

The secondary $\mathrm{CHs}$ can be selected from the primary $\mathrm{CHs}$ and are elected on the basis of probability from those nodes which had already become the primary $\mathrm{CHs}$. The primary $\mathrm{CHs}$ only can choose the secondary CHs. They check distance between each other and the ones that are at minimum distance from them are selected the secondary CHs. It also uses advance nodes and normal nodes. The process of selecting the primary $\mathrm{CH}$ is same as in Sep by generating a rando0m number between 0 and 1 and then comparing it with the threshold value. These primary $\mathrm{CHs}$ then aggregate data collected from other nodes and transmit it to the secondary $\mathrm{CHs}$ which further send it to the BS. Thus, minimizing the transmission distance between the secondary $\mathrm{CHs}$ and the BS results in less consumption of energy.

Thus HSEP outperforms the other protocols because it is based on clustering hierarchy in which $\mathrm{CHs}$ are of two levels. This hierarchical clustering reduces the transmission distance 
and hence results in less power dissipation. Also the stability period of HSEP is higher as compared to others.

Thus, energy heterogeneity should be one of the key factors to be considered when designing a robust protocol for WSN. The goal is to design a modified protocol that is more robust and can ensure longer network lifetime while taking performance measures into consideration.

\section{(g)Energy Consumption Rate based Stable Election Protocol (ECRSEP)}

Clustered sensor networks may be homogeneous or heterogeneous sensor networks. Homogeneous networks are those in which all nodes have same amount of energy. Owing to static clustering in homogeneous networks, the $\mathrm{CHs}$ will be overloaded with long distance transmissions and need extra energy for processing. This results into the death of $\mathrm{CHs}$ before the other nodes. So, to ensure that all no0des die at the same time, a small amount of residual energy is wasted. One way to do this is to rotate the $\mathrm{CH}$ periodically and randomly over all nodes. In heterogeneous networks two or more different types of nodes are used based on their initial energy levels.

In ECRSEP, $\mathrm{CH}$ selection is based on the energy consumption rate (ECR).

It is defined as the ECR $=\frac{E_{\text {int }}-E_{r}}{\gamma-1}$, where $E_{\text {int }}$ is the initial energy, $E_{y}$ is the residual energy and $r$ is the current round. The $\mathrm{CH}$ selection in the next round is based on the ECR of the previous round. A node with less ECR in the previous round is selected as $\mathrm{CH}$ in the next round.

Let $n$ be the number of rounds to become $\mathrm{CH}$ for nodes $\mathrm{S}$. we call it the rotating epoch. Let $p=\frac{1}{n}$ is average probability to become $\mathrm{CH}$ during $\mathrm{n}$ rounds.

We have, $\mathrm{p}=$ popt $^{*}$ ECR

Total number of CHs per epoch is $\sum_{\mathrm{i}}^{N} P_{\mathrm{i}}=\mathrm{N}$ popt

In two level heterogonous networks, $p_{0 p t}$ is replaced by weighted probabilities of advance and normal nodes

$$
\begin{aligned}
& P_{a d v}=\frac{P_{o p t} \frac{E_{i}-E_{r}}{r-1}}{1+a m} \\
& P_{\text {nrm }}=\frac{P_{o p t}[1+a) \frac{E_{1}-E_{r}}{r-1}}{1+a m}
\end{aligned}
$$

The results show that ECRSEP has enhanced stability period and network life than other protocols. ECRSEP achieves maximum lifetime of the network. LEACH goes sooner to unstable as it is very sensitive to heterogeneity. SEP extend the stability period by assigning probabilities of $\mathrm{CH}$ election weighted by relative initial energy. ESEP has three levels of heterogeneity so it has longer stability period than SEP. HSEP uses hierarchical clustering approach and thus reduces the transmission energy. And above all ECRSEP outperforms all of them.

\section{(h) HEER}

HEER [12] is defined as Hybrid Energy Efficient Reactive Protocol for Wireless Sensor Networks. In HEER the Cluster Head $(\mathrm{CH})$ selection is based on the ratio of residual energy of node and average energy of network. Moreover, to conserve more energy, it introduces the Hard Threshold (HT) and Soft Threshold (ST). Finally, the simulation result shows that the protocol has not only prolonged the network lifetime but also significantly increased stability period. It improves the stable region for clustering hierarchy process for a reactive network in homogeneous and heterogeneous environment. It uses the initial and residual energies of the nodes to become Cluster Head i.e. similar to that of DEEC (Distributed energy efficient clustering protocol) .It does not require any global knowledge of energy at any election round. The $\mathrm{CH}$ transmits two threshold values when cluster formation is completed, i.e. $H T$ and $S T$. The nodes sense their environment repeatedly and if a parameter from the attributes set reaches its HTvalue, the node switches on its transmitter and transmits data.

Important Features:

1) HEER performs best for time critical applications in both homogeneous and heterogeneous environment.

2)It reduces the number of transmissions resulting in the reduction of energy consumption.

3)It increases the stability period and network lifetime.

\section{RELATED WORK}

Due to small batteries [1] in WSNs, the optimal and efficient usage of energy is an important factor. To enhance the life time of the network, clustering is the most efficient technique. In ECRSEP, the CHs are selected based on the weighted election probabilities of each node according to the Energy Consumption Rate (ECR) of each node. But its limitation is that all nodes should be capable of becoming a $\mathrm{CH}$. Each node in TSEP [2] maintains a routing table which keeps record of the destinations and the number of hops needed to reach the destination. The nodes keep sensing the network for any kind of changes in the system can communicate the data sensed to the other nodes in the network. The reactive routing protocols, unlike the proactive, do not sense the network on a regular basis. The constant updating of routing table is not required. This is on-demand protocol which means that the nodes search for route when needed and then transmit the data. TSEP is a reactive protocol which uses three levels of heterogeneity. These protocols respond instantly to the changes in the parameters of the network. The transmission of data consumes more energy than sensing and takes place only when a certain threshold is achieved.

HSEP [3] protocol is heterogeneous-aware in the sense that the election probabilities of nodes to become $\mathrm{CHs}$ are weighted by their initial energy relative to other nodes in the network which enlarge the time gap before the death of first node in the system thus increasing the stability period. As the distance between the cluster head and sink increases the power consumption is also greatly increased. HSEP reduces the transmission cost from $\mathrm{CH}$ to base station by considering two types of cluster hierarchies: primary and secondary $\mathrm{CHs}$.

The new LEACH-N [4] protocol modifies the process of $\mathrm{CH}$ selection by adding the factor of residual energy while calculating $\mathrm{T}(\mathrm{n})$ which is

$$
\mathrm{T}(\mathrm{n})=\left\{\begin{array}{c}
\frac{\mathrm{p}}{1-\mathrm{p} v\left(\mathrm{r} \text { mod } \frac{1}{p}\right)} * \frac{E_{\text {init }}-E_{\text {currmat }}}{E_{\text {init }}} \\
0, \quad \text { otherwise }
\end{array}, \mathrm{n} \in \mathrm{G}\right.
$$

$E_{\text {init }}$ is the initial energy, $E_{\text {current }}$ is the node's current residual energy. By applying this technique, the nodes who have consumed more energy will have less probability to become $\mathrm{CH}$ again. This ensures optimized way of $\mathrm{CH}$ 
selection and prolongs the network lifetime. The residual factor thus, reduces the energy cost of network nodes.

The $\mathrm{CH}$ [5] node in the network broadcast to the radium $\mathrm{R}$ and forms the measuring area. The nods in the measuring area reflect back the signals to $\mathrm{CH}$. The broadcasting radium $\mathrm{R}$ measures the nearby node distribution. The total measuring area of $\mathrm{CH}$ reaches a cover rate, such that the node distribution in measuring area reflects node distribution in clustering area. Also, as the $\mathrm{R}$ increases, the overlap rate also increases. This leads to increase in energy consumption. The node which has low node density if selected the $\mathrm{CH}$, the other nodes need to travel a long distance for communication.

The ESEP [7] is a protocol for cluster-head selection in a hierarchical clustered heterogeneous network. The authors have assumed that the nodes are static and are distributed randomly in the network and the dimensions of the sensor field and sink are known. SEP was based on weighted election probabilities of each node to be a cluster head as per their energies. It considered two types of nodes: advance nodes and normal nodes with advance nodes having high amount of initial energy than the normal nodes of the network.

In SCHE [8], the nodes will always have some data to transmit to the base station or sink. It also uses the concept of data aggregation to avoid overload of information or the access to data. It obtains the stable probability to select the $\mathrm{CH}$. An optimum value of probability is obtained which helps to select a $\mathrm{CH}$. The simulations were performed to evaluate the energy consumption by LEACH and SCHE. For the same transmission distance between the base station and other nodes, the results show that SCHE consumes less energy as compared to LEACH due to stable cluster head election probability whereas LEACH applies random cluster head selection

SEP [9] assumes that the base station is not energy constrained compar3ed to the nodes of the system. Also the nodes are assumed to be uniformly distributed over the field . SEP uses two types of nodes based on their energy level. The nodes that have more energy relative to other nodes are referred as advanced nodes and the other nodes are normal nodes. The clustering hierarchy of LEACH protocol is applied to heterogeneous environment with differing energy levels among the nodes. SEP attempts to maintain the energy consumption of the network by considering advance nodes that have higher energy, to become cluster-heads more often than the normal nodes.

By adding a factor of advance nodes, it is observed that the total energy of the system increases by a factor $(1+\alpha) . m$ where $\alpha$ is the additional energy factor between advance and normal nodes and $m$ is the fraction of advance nodes in the system. The stable region could also be increased by that factor if each normal and advance node becomes a cluster-head once each round of an epoch. The simulation results show that the SEP protocol is successful in extending the stable region by assigning probabilities weighted by the initial energies of the nodes. And due to increased stability, the throughput of SEP is also higher than LEACH protocol.

TEEN [10] is as reactive routing protocol as it responds immediately to the changes in the value of certain attributes. Each time a cluster changes, the cluster-head broadcasts the parameters to its members. It defines hard and soft thresholds. Hard threshold is a value beyond which the node sensing this value must turn on its radio and report to its $\mathrm{CH}$. A soft threshold is a small change in the value of sensed attribute that initiates the node to switch on its radio and transmit. The hard threshold tries to reduce the number of transmissions by allowing the nodes to transmit only when the sensed attribute is in the range of interest. The soft threshold reduces the number of transmissions which might have occurred when there is a small change in the value of sensed attribute. TEEN is useful for time-critical applications where the data reaches the user immediately. The energy consumption is reduced as the nodes sense data only when there is a sudden change in the value of attributes.

LEACH [11] is a self-organizing protocol that uses randomization to select the cluster base stations to distribute the load evenly among the other nodes in the network. The LEACH protocol selects $\mathrm{CHs}$ in two phases: set up phase and steady phase. The nodes organize themselves into local clusters with a cluster-head $(\mathrm{CH})$ node. The high energy cluster-head nodes are randomly rotating among the other sensors so that the battery of just a single sensor is not drained. These $\mathrm{CHs}$ broadcast their status to other sensor nodes and then these nodes decide as to join which $\mathrm{CH}$. When all the nods have chosen their respective clusters, the $\mathrm{CH}$ prepares a schedule for its nodes.

\section{GAPS IN LITERATURE}

The survey on different WSNs has been done by considering the various stable election based protocols like

\section{SEP, ESEP, ECRSEP etc.}

1. SEP, ESEP and ECRSEP continues to punish advance and intermediate nodes i.e. no special protection for over utilization of advance and intermediate nodes.

2. SEP, ECRSEP and ESEP has also neglected the use of thresholding i.e. hard and soft thresholding to reduce the energy consumption.

3. It has been found that the most of the existing researchers have neglected the use of absolute residual energy level (ARL) value to avoid this unbalanced case in three-level heterogeneous network and to save intermediate and advance nodes from over penalized.

\section{CONCLUSION AND FUTURE WORK}

Wireless Sensor Networks (WSNs) are composed of multiple unattended ultra-small, limited-power sensor nodes. These nodes have partial processing, power resource capabilities and wireless communication, which send sensed data to sink or Base Station (BS). A novel technique as efficient Cluster head Replacement has been proposed i.e. HEER. In this, the Cluster Head $(\mathrm{CH})$ selection is based on the ratio of residual energy of node and average energy of network. Moreover, to conserve more energy, HEER has also utilized the Hard Threshold (HT) and Soft Threshold (ST). But HEER does not use the inter cluster data aggregation, but it is not deterministic in nature, as cluster head selection is based upon the weighted probabilities.

So to overcome this problem a deterministic approach will be proposed to enhance the cluster head selection. The idea behind deterministic cluster head selection is simple; firstly all the nodes will be sorted based upon their residual energies top $10 \%$ nodes will be selected as cluster head. So the selection criteria will have deterministic decisions because node with 
highest first energy will become cluster head than the probability. It will increase the overall network lifetime.

\section{REFERENCES}

[1] Rehman, O., Javaid, N., Manzoor, B., Hafeez, A., Iqbal, A., \&Ishfaq, M. (2013). Energy Consumption Rate based Stable Election Protocol (ECRSEP) for WSNs. Procedia Computer Science, 19, 932-937.

[2] Kashaf, A., Javaid, N., Khan, Z. A., \& Khan, I. A. (2012, December). TSEP: Threshold-sensitive Stable Election Protocol for WSNs. In Frontiers of Information Technology (FIT), 2012 10th International Conference on (pp. 164-168). IEEE.

[3] Khan, A. A., Javaid, N., Qasim, U., Lu, Z., \& Khan, Z. A. (2012). HSEP: Heterogeneity-aware Hierarchical Stable Election Protocol for WSNs. arXiv preprint arXiv:1208.2335.

[4] Li, Yuling, Luwei Ding, and Feng Liu. "The improvement of LEACH protocol in WSN." Computer Science and Network Technology (ICCSNT), 2011 International Conference on. Vol. 2. IEEE, 2011.

[5] Peng, J., Chengdong, W., Yunzhou, Z., \&Fei, C. (2011, September). A Low-Energy Adaptive Clustering Routing Protocol of Wireless Sensor Networks. InWireless Communications, Networking and Mobile Computing (WiCOM), 2011 7th International Conference on (pp. 14). IEEE.

[6] Aderohunmu, Femi A., and Jeremiah D. Deng. "An Enhanced Stable Election Protocol (and Heterogeneous WSN." XH Wu, S. Wang," Performance comparison of
LEACH on LEACH-C protocols by NS2," Proceedings of 9th International Symposium SEP) for Clustered Distributed Computing and Applications to Business, Engineering and Science. Hong Kong, China. 2010.

[7] Islam, M. M., Matin, M. A., \&Mondol, T. K. (2012, June). Extended Stable Election Protocol (SEP) for threelevel hierarchical clustered heterogeneous WSN. In Wireless Sensor Systems (WSS 2012), IET Conference on (pp. 1-4). IET.

[8] Muhamad, W. N. W., Dimyati, K., Mohamad, R., Haron, M. A., Sarnin, S., Wahab, N., \& Aziz, N. H. A. (2008, December). Evaluation of Stable Cluster Head Election (SCHE) routing protocol for wireless sensor networks. In RF and Microwave Conference, 2008. RFM 2008. IEEE International (pp. 101-105). IEEE.

[9] Smaragdakis, Georgios, Ibrahim Matta, and AzerBestavros. SEP: A stable election protocol for clustered heterogeneous wireless sensor networks. Boston University Computer Science Department, 2004.

[10] Manjeshwar, Arati, and Dharma P. Agrawal. "TEEN: ARouting Protocol for Enhanced Efficiency in Wireless Sensor Networks." IPDPS. Vol. 1. 2001.

[11] Heinzelman, Wendi Rabiner,Anantha Chandrakasan and HariBalakrsihnan. "Energy-efficient communication protocol for wireless microsensor netweorks". System Sciences, 2000. Proceedings of the 33rd Annual Hawaii International Conference on. IEEE, 2000.

[12] N. Javaid, S. N. Mohammad, K. Latif, U. Qasim, Z. A. Khan, M. A. Khan."HEER; Hybrid energy efficient reactive protocol" IEEE, August 2013. 